\title{
Trouver, tester, « tracer » et isoler énergiquement pour battre la COVID-19
}

\author{
Larissa M. Matukas MSc MD, Irfan A. Dhalla MD MSc, Andreas Laupacis MD MSc
}

Citation : CMAJ 2020 October 5;192:E1164-5. doi : 10.1503/cmaj.202120-f; diffusion hâtive le 9 septembre 2020

Voir la version anglaise de l'article ici : www.cmaj.ca/lookup/doi/10.1503/cmaj.202120; voir la recherche connexe en anglais ici : www. cmaj.ca/lookup/doi/10.1503/cmaj.201128; voir la recherche connexe en français ici : www.cmaj.ca/lookup/doi/10.1503/cmaj.201128-f

A u Canada, environ 300 personnes ont été déclarées positives au coronavirus du syndrome respiratoire aigu sévère 2 (SRAS-CoV-2) chaque jour à la fin d'août 2020, ce qui correspond à environ $20 \%$ de la moyenne quotidienne rapportée à la mi-avril ${ }^{1}$. Toutefois, lors d'un déconfinement effectué alors que le virus est encore présent dans la communauté, le nombre d'infections augmente habituellement, comme on l'a vu ailleurs dans le monde et comme le suggère la graduelle augmentation des infections au Canada depuis la mijuillet $^{1}$. Une forte hausse des taux d'infections au SRAS-CoV-2 compromettra la santé, l'éducation et le gagne-pain des gens. Comment peut-on réduire ce risque?

Étant donné que le SRAS-CoV-2 est transmis par des personnes infectées présentant ou non des symptômes, pour être efficace, une stratégie doit reposer sur le dépistage de toutes les personnes infectées.

Premièrement, les campagnes de la santé publique doivent être claires et cohérentes, et insister sur le fait que quiconque présente des symptômes compatibles avec ceux de la maladie à coronavirus 2019 (COVID-19) doit se faire tester immédiatement et se mettre en quarantaine en attendant les résultats. Cela contraste nettement avec les conseils donnés précédemment pour d'autres maladies respiratoires qui demandaient aux gens de rester à la maison.

Deuxièmement, étant donné que les personnes infectées asymptomatiques contribuent aussi et significativement à la propagation du SRAS-CoV- $2^{2}$, les campagnes de la santé publique doivent continuer de marteler ses consignes sur le lavage des mains, le port du couvre-visage, la distanciation physique et le respect des petites bulles sociales. Les personnes asymptomatiques qui sont infectées par le SRAS-CoV-2 doivent aussi être identifiées pour pouvoir s'isoler avant de propager le virus ${ }^{3,4}$. Mais parmi les personnes asymptomatiques, lesquelles sont les plus susceptibles d'avoir contracté le SRAS-CoV- $2^{5}$ ?

Dans une recherche publiée par le CMAJ, Campbell et ses collaborateurs ont estimé les coûts associés à diverses stratégies de dépistage auprès de groupes susceptibles d'être plus à risque à l'égard de l'infection : ceux qui ont été en contact avec des gens ayant un diagnostic récent d'infection au SRAS-CoV-2, la maind'œuvre essentielle qui ne peut pas travailler à domicile, les travailleurs de la santé, les personnes qui vivent et travaillent dans des établissements de soins de longue durée et les enfants et professeurs dans les écoles 5 . Nous appuyons fortement l'une des stratégies décrites, soit tracer et tester énergiquement les contacts de personnes récemment diagnostiquées, qui s'accompagne d'un coût estimé de 238 millions de dollars supplémentaires par année ${ }^{5}$, un montant dérisoire si on le compare à la réponse fiscale des gouvernements fédéral et provinciaux face à la pandémie à ce jour et aux pertes économiques accumulées par suite de la pandémie au Canada.

Pour être efficace, une stratégie énergique de dépistage et de traçage doit disposer de ressources permettant d'identifier et de tester de manière proactive tous les contacts proches d'individus porteurs d'un diagnostic récent d'infection au SRAS-CoV-2, les résultats des tests doivent être disponibles rapidement et il faut réduire le fardeau imposé aux personnes forcées de s'isoler.

Il ne sera possible d'obtenir plus rapidement (en moins de 24 heures) les résultats une fois les spécimens recueillis que si ces derniers sont acheminés sans délai des points de collecte aux laboratoires, et que si les laboratoires travaillent à l'analyse des échantillons 24 heures sur 24, 7 jours sur 7 . Toute l'information allant du premier contact de la personne avec le point de collecte jusqu'à la transmission des résultats aux patients, aux professionnels de la santé et aux responsables de la santé publique doit être gérée électroniquement pour optimiser l'efficience de l'approche «trouver, tester, tracer et isoler».

Certains individus auront de la difficulté à s'isoler puisqu'ils doivent gagner leur vie, que leur situation de vie les en empêche (p. ex., vivre à 5 dans un logement ne comptant qu'une seule salle de bains), ou qu'ils doivent s'occuper d'autres personnes. Les gouvernements doivent s'assurer que les employés qui obtiennent un résultat positif au dépistage du SRAS-CoV-2 puissent continuer de recevoir leur salaire tout en s'isolant, que des établissements, comme des hôtels réservés, soient facilement et 
gratuitement accessibles aux personnes qui ne peuvent s'isoler dans leur milieu de vie, et que d'autres formes de soutien soient mis à leur disposition (p. ex., épicerie).

La préparation de rapports est nécessaire, tant à des fins d'amélioration de la qualité que d'imputabilité; ils serviront aussi à dégager les priorités. La plupart des provinces font actuellement état du nombre de nouveaux cas d'infections au SRASCoV-2, du nombre de tests effectués chaque jour et de certaines données démographiques sur les personnes positives ${ }^{6,7}$. Mais cela ne suffit pas. Les rapports doivent aussi inclure le nombre de contacts étroits identifiés quotidiennement, le nombre de contacts testés et mis en quarantaine avec succès et l'intervalle entre le prélèvement des échantillons et l'obtention des résultats. D'autres pays, comme la Nouvelle-Zélande et l'Australie, soulignent dans leurs comptes-rendus quotidiens le nombre de nouveaux cas n'ayant aucun lien épidémiologique connu ${ }^{8,9}$. Au Canada, les gouvernements devraient aussi déclarer ces chiffres; cela aiderait à mettre vraiment l'accent sur l'identification et l'isolement du plus grand nombre possible de contacts des personnes infectées.

Les tests seuls ne préviennent pas l'infection au SRAS-CoV-2, mais réduire les contacts avec des gens infectés, oui. Par contre, le diagnostic et la mise en quarantaine rapides des personnes infectées au SRAS-CoV-2, symptomatiques ou non, et le fait de tracer, tester et isoler énergiquement les contacts permettront d'assurer la sécurité des écoles, des milieux de travail et des collectivités au Canada. Il est temps que nos gouvernements investissent massivement pour « trouver, tester, tracer et isoler».

\section{Références}

1. Areas in Canada with cases of COVID-19 as of September 7, 2020 [table]. In: Coronavirus disease 2019 (COVID-19): epidemiology update. Ottawa: Public Health Agency of Canada; updated 2020 Sept. 7. Accessible ici : https://health -infobase.canada.ca/covid-19/epidemiological-summary-covid-19-cases. html\#dataTable (consulté le 8 septembre 2020).
2. Furukawa NW, Brooks JT, Sobel J. Evidence supporting transmission of severe acute respiratory syndrome coronavirus 2 while presymptomatic or asymptomatic. Emerg Infect Dis. 2020;26:e201595.

3. Hellewell J, Abbott S, Gimma A, et al. Feasibility of controlling COVID-19 outbreaks by isolation of cases and contacts. Lancet Glob Health. 2020;8:e488-96.

4. Ng V, Fazil A, Waddell LA, et al. Projected effects of nonpharmaceutical public health interventions to prevent resurgence of SARS-CoV-2 transmission in Canada. CMAJ 9 août 2020. [Cyberpublication avant impression]. doi: 10.1503/cmaj.200990.

5. Campbell JR, Uppal A, Oxlade O, et al. Active testing of groups at increased risk of acquiring SARS-CoV-2 in Canada: costs and human resource needs. CMAJ 9 septembre 2020. [Cyberpublication avant impression]. doi: 10.1503/cmaj. 201128.

6. Provincial Health Services Authority; BC Centre for Disease Control. British Columbia COVID-19 Dashboard. ArcGIS [logiciel]. Accessible ici : experience.arcgis.com/ experience/a6f23959a8b14bfa989e3cda29297ded (consulté le 3 septembre 2020).

7. Ontario COVID-19 data tool. Toronto: Public Health Ontario. Accessible ici : www.publichealthontario.ca/en/data-and-analysis/infectious-disease/covid-19 -data-surveillance/covid-19-data-tool (consulté le 3 septembre 2020).

8. Coronavirus (COVID-19) current situation and case numbers [news]. Canberra $(\mathrm{AU})$ : Australia Australian Government Department of Health. Accessible ici : www.health.gov.au/news/health-alerts/novel-coronavirus-2019-ncov-health -alert/coronavirus-covid-19-current-situation-and-case-numbers (consulté le 23 septembre 2020).

9. Managed isolation and quarantine. In: COVID-19: current cases. Wellington (New Zealand): Ministry of Health. Accessible ici : www.health.govt.nz/our-work /diseases-and-conditions/covid-19-novel-coronavirus/covid-19-current-situation /covid-19-current-cases\#managed-isolation (consulté le 3 septembre 2020).

Intérêts concurrents : Voir www.cmaj.ca/site/misc/cmaj_staff. xhtml pour Andreas Laupacis. Aucun autre intérêt concurrent déclaré.

Affiliations : Départements de médecine de laboratoire et pathobiologie et de médecine (Matukas), Université de Toronto; Division de microbiologie (Matukas), Départements de médecine de laboratoire et de médecine, Hôpital St. Michael's, Unity Health Toronto, Département de médecine et Institut des politiques, de la gestion et de l'évaluation de la santé (Dhalla), Université de Toronto; Unity Health Toronto (Dhalla), Toronto, Ont.; rédacteur en chef (Laupacis), CMAJ.

Correspondance : Rédaction du CMAJ, cmaj@cmajgroup.ca 